\section{Shape Parameter for a Non-Axisymmetric Isothermal Dendrite}

\section{G. B. McFadden S. R. Coriell}

U.S. DEPARTMENT OF COMMERCE Technology Administration National Institute of Standards and Technology

100 Bureau Drive Stop 8910

Gaithersburg, MD 20899-8910, USA

\section{R. F. Sekerka}

University Professor

Physics and Mathematics

Carnegie Mellon University

Pittsburgh, PA 15213-3890, USA

QC 



\section{Shape Parameter for a}

Non-Axisymmetric Isothermal

Dendrite

\section{G. B. McFadden \\ S. R. Coriell}

U.S. DEPARTMENT OF COMMERCE Technology Administration National Institute of Standards and Technology

100 Bureau Drive Stop 8910

Gaithersburg, MD 20899-8910, USA

\section{R. F. Sekerka}

University Professor

Physics and Mathematics

Carnegie Mellon University

Pittsburgh, PA 15213-3890, USA

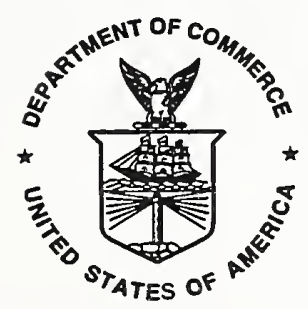

U.S. DEPARTMENT OF COMMERCE William M. Daley, Secretary

TECHNOLOGY ADMINISTRATION Gary R. Bachula, Acting Under Secretary for Technology

NATIONAL INSTITUTE OF STANDARDS

AND TECHNOLOGY

Raymond G. Kammer, Director 



\title{
Shape Parameter for a Non-Axisymmetric Isothermal Dendrite
}

\author{
G. B. McFadden ${ }^{a, *}$, S. R. Coriell ${ }^{a}$, R. F. Sekerka ${ }^{b}$ \\ ${ }^{a}$ National Institute of Standards and Technology \\ Gaithersburg, MD 20899-8910, USA \\ ${ }^{b}$ University Professor, Physics and Mathematics \\ Carnegie Mellon University \\ Pittsburgh, PA 15213-3890, USA
}

\begin{abstract}
In previous work, we found approximate solutions for paraboloids having perturbations with four-fold axial symmetry in order to model dendritic growth in cubic materials. These solutions provide self-consistent corrections through second order in a shape parameter $\epsilon$ to the Peclet number - supercooling relation of the Ivantsov solution. The parameter $\epsilon$ is proportional to the amplitude of the four-fold correction to the dendrite shape, as measured from the Ivantsov paraboloid of revolution. We calculate $\epsilon$ by comparing the dendrite tip shape to the portion of the equilibrium shape near the growth direction, [001], for anisotropic surface free energy of the form $\gamma=\gamma_{0}\left[1+4 \epsilon_{4}\left(n_{x}^{4}+n_{y}^{4}+n_{z}^{4}\right)\right]$, where the $n_{i}$ are components of the unit normal of the crystal surface. This comparison results in $\epsilon=-2 \epsilon_{4}$, independent of the Peclet number. From the experimental value of $\epsilon_{4}$, we find $\epsilon \approx-0.011$, in good agreement with the measured value $\epsilon \approx-0.008$ of LaCombe et al.
\end{abstract}

PACS: 81.10.Aj, 64.70.Dv, 66.10.Cb, 81.10.Mx

Keywords: dendritic growth, supercooled liquids, non-axisymmetric dendrites, Ivantsov relation, anisotropic surface free energy

${ }^{*}$ Corresponding author. Fax + $1301990-4127$; e-mail: mcfadden@nist.gov 


\section{Introduction}

In a previous paper [1] we calculated the correction to the relationship between the Peclet number $P$ and the dimensionless supercooling, $S$, for a non-axisymmetric isothermal dendrite growing from a pure supercooled melt. For four-fold axial symmetry, the dendrite shape in cylindrical coordinates $(r, \phi, z)$ is of the form

$$
\frac{z}{\rho}=\frac{1}{2}-\frac{1}{2}\left(\frac{r}{\rho}\right)^{2}-\frac{\epsilon}{2} \cos 4 \phi\left(\frac{r}{\rho}\right)^{4}+\frac{\epsilon^{2}}{2}\left[\alpha(P)\left(\frac{r}{\rho}\right)^{4}+\beta(P)\left(\frac{r}{\rho}\right)^{6}\right]+O\left(\epsilon^{3}\right),
$$

where the shape parameter $\epsilon$ represents the amplitude of the four-fold perturbation to the axisymmetric paraboloid, and $\rho$ is the radius of curvature of the dendrite tip. Specifically, $P=V \rho / 2 \kappa$ and $S=c_{V}\left(T_{M}-T_{\infty}\right) / L_{V}$, where $V$ is the dendrite growth speed, $\kappa$ is the thermal diffusivity of the melt, $c_{V}$ is heat capacity per unit volume, $L_{V}$ is the latent heat per unit volume, $T_{M}$ is the melting point, and $T_{\infty}$ is the far-field temperature of the supercooled melt. The corresponding correction to the $P-S$ relation is found to have the form

$$
S=P e^{P} E_{1}(P)+\frac{\epsilon^{2}}{2} S^{(2)}(P)+O\left(\epsilon^{3}\right)
$$

The specific dependence of the coefficients $\alpha$ and $\beta$, and the correction $S^{(2)}$, on Peclet number are worked out in detail in Ref. [1]. Here, the function $E_{1}$ is the exponential integral [2]. For $\epsilon=0$ this yields the well-known result of Ivantsov [3]. Other researchers have also noted that the first-order term proportional to $r^{4} \cos 4 \phi$ is consistent with an isothermal solution that has been employed in microscopic solvability theory [4-7].

Based on the experimental measurements of LaCombe et al [8], for succinonitrile (SCN) at $P \approx 0.004$, we estimated a value of $\epsilon \approx-0.008$, with the convention that $\phi=0$ corresponds to the $[100]$ direction. The corresponding correction to $S$ was about a $9 \%$ increase, in general agreement with the experimental results [8-10].

In this paper, we estimate the shape parameter $\epsilon$ theoretically on the basis of a simple idea, namely, that the shape of the isothermal but anisotropic dendrite tip is approximately the same as a portion of the equilibrium shape of an isothermal body with slightly anisotropic surface free energy. For a cubic crystal, such as SCN, we assume a surface free energy $\gamma(\widehat{\mathbf{n}})$ 
of the form

$$
\gamma=\gamma_{0}\left[1+4 \epsilon_{4}\left(n_{x}^{4}+n_{y}^{4}+n_{z}^{4}\right)\right]
$$

where $\gamma_{0}$ and $\epsilon_{4}$ are constants, and $\widehat{\mathbf{n}}=\left(n_{x}, n_{y}, n_{z}\right)$ is the unit normal of the crystal surface. This corresponds to the leading order expansion of $\gamma$ in spherical harmonics compatible with cubic symmetry; the next non-vanishing term is of sixth degree in $\widehat{\mathbf{n}}$. In the subsequent analysis, we will assume $\left|\epsilon_{4}\right| \ll 1$ and neglect all higher order contributions of $\epsilon_{4}[11,12]$. We note that the equilibrium shape is a closed convex body in a strictly isothermal environment, whereas our dendrite model [1] corresponds to a semi-infinite body with an isothermal surface that is growing from a non-isothermal melt. For small supercoolings, however, we expect the dendrite tip shape to be similar to the portion of the equilibrium shape near the growth direction, which is [001] for SCN.

\section{Analysis}

It is well-known that for small anisotropy, the equilibrium shape is geometrically similar to a polar plot of the surface free energy [12-15]. Thus the equilibrium shape can be written in the form

$$
\frac{r_{s}}{R}=1+4 \epsilon_{4}\left[\cos ^{4} \Theta+\sin ^{4} \Theta\left(\frac{3}{4}+\frac{1}{4} \cos 4 \Phi\right)\right]+O\left(\left|\epsilon_{4}\right|^{2}\right)
$$

where $\mathbf{r}_{\mathbf{s}}$ is the position vector of the equilibrium shape, $r_{s}=\left|\mathbf{r}_{\mathbf{s}}\right|, R$ is a constant scale factor, and $\Theta$ and $\Phi$ are the spherical angles of the unit normal, so that $\widehat{\mathbf{n}}=(\sin \Theta \cos \Phi, \sin \Theta \sin \Phi, \cos \Theta)$. Furthermore, to first order in the anisotropic term, $\Theta$ and $\Phi$ can be replaced by the angles $\theta$ and $\phi$ that specify the orientation of the vector $\mathbf{r}_{\mathbf{s}}$. Thus a polar plot of the equilibrium shape has the form

$$
\frac{r_{s}(\theta, \phi)}{R}=1+4 \epsilon_{4}\left[\cos ^{4} \theta+\sin ^{4} \theta\left(\frac{3}{4}+\frac{1}{4} \cos 4 \phi\right)\right]+O\left(\left|\epsilon_{4}\right|^{2}\right) .
$$

We proceed to write this expression in terms of cylindrical coordinates to compare with 
Eq. (1). Using $r_{s}=\sqrt{r^{2}+z^{2}}, \cos \theta=z / \sqrt{r^{2}+z^{2}}$ and $\sin \theta=r / \sqrt{r^{2}+z^{2}}$, we have

$$
\frac{\sqrt{r^{2}+z^{2}}}{R}=1+\frac{4 \epsilon_{4}}{\left(r^{2}+z^{2}\right)^{2}}\left[z^{4}+r^{4}\left(\frac{3}{4}+\frac{1}{4} \cos 4 \phi\right)\right]+O\left(\left|\epsilon_{4}\right|^{2}\right) .
$$

Near the [001] direction, $|r / z| \ll 1$, so we can expand Eq. (6) to obtain

$$
\frac{z}{R}=1+4 \epsilon_{4}-\frac{1}{2} \frac{r^{2}}{R^{2}}\left(1+12 \epsilon_{4}\right)-\frac{1}{8} \frac{r^{4}}{R^{4}}\left(1-36 \epsilon_{4}\right)+\epsilon_{4} \frac{r^{4}}{R^{4}} \cos 4 \phi+O\left(\left|\epsilon_{4}\right|^{2},(r / R)^{6}\right)
$$

In order to compare Eqs. (1) and (7), we first recognize that the origin of $z$ is arbitrary, so that the constant terms may be ignored. Multiplication of Eq. (7) by $R / \rho$ and comparison of the term in $r^{2}$ with the corresponding term in Eq. (1) shows that $R=\rho\left(1+12 \epsilon_{4}\right)+O\left(\left|\epsilon_{4}\right|^{2}\right)$. Then comparison of the terms in $\cos 4 \phi$ yields our central result,

$$
\epsilon=-2 \epsilon_{4}+O\left(\left|\epsilon_{4}\right|^{2}\right)
$$

We note that the the axisymmetric term proportional to $r^{4}$ in Eq. (7) has no counterpart in Eq. (1). This arises because the equilibrium shape is a closed convex body, whereas the dendrite is a semi-infinite body. The closure of this equilibrium shape is described properly by Eqs. (4)-(6), but is lost once one resorts to the expansion in Eq. (7).

\section{Discussion}

The anisotropy of the surface free energy for SCN has been measured by Glicksman and Singh [11] and Muschol et al. [12], resulting in $\epsilon_{4}=0.0055 \pm 0.0015$, which from Eq. (8) yields $\epsilon=-0.011 \pm 0.003$. This compares favorably with the direct measurements of LaCombe et al. [8] which result in $\epsilon \approx-0.008$. Note, however, that the experimental determination of $\epsilon$ is based on measurements of the dendrite shape for distances of up to ten tip radii from the tip, whereas our comparison to the equilibrium shape is only valid within a fraction of a tip radius from the tip. Another theoretical estimate of $\epsilon$ has been made by Brener et al. $[6,7]$ based on microscopic solvability theory, and, in our notation, results in $|\epsilon|=1 / 48 \approx 0.02$, which is about a factor of two larger than the experimental value. Their result is independent of $\epsilon_{4}$. 
By means of numerical computations based on a phase-field model, Karma and Rappel [16] calculated a shape anisotropy for $S=0.45$ and an effective surface free energy anisotropy of 0.0066 , resulting in $|\epsilon|=0.019$, close to the value of Brener et al. Karma and Rappel find that $|\epsilon|$ increases for larger values of the effective anisotropy.

A value of $\epsilon_{4}=0.025$ has been measured for pivalic acid [12]. This anisotropy is about five times larger than that of SCN. No measurements of the actual shape anisotropy are yet available, but we caution that this value of $\epsilon_{4}$ might be too large for our expansion to be valid. One could, however, extend the equilibrium shape to higher order in $\epsilon_{4}$, which would also delineate the range of validity of the linear expansion.

Note that the value of $\epsilon$ given by Eq. (8) is independent of the Peclet number $P$. This is supported by preliminary measurements by LaCombe [17] over a limited range of supercoolings. Accordingly, in Fig. 1 we plot the value of $S$ from Eq. (2) for $\epsilon=-0.008$. For the smaller values of $P$ in the figure, our corrections to $S$ are too large for our expansion in $\epsilon$ to be valid, resulting in a nearly vertical curve near $P=0.001$. In the range $0.004<P<0.01$, our results resemble the experimental values measured by Koss et al., which also lie slightly below the Ivantsov curve (see Fig. 6 of Ref. [9]). For $P$ much below 0.004, the experimental data actually lie above the Ivantsov curve, possibly due to the effects of finite container size and/or convection $[18,19]$. Thus, the effects of non-axisymmetry versus those due to finite container sizes and/or convection tend to affect $S$ in an opposing manner.

\section{Acknowledgments}

The authors are grateful to S.A. Langer and R.J. Schaefer for helpful discussions. This work was conducted with the support of the Microgravity Research Division of NASA. RFS is also grateful for support of the National Science Foundation, Grant DMR9634056.

\section{References}

[1] G.B. McFadden, S.R. Coriell, R.F. Sekerka, submitted to the Journal of Crystal Growth, 1999. 
[2] M. Abramowitz, I. A. Stegun, Handbook of Mathematical Functions, Applied Mathematics Series 55, National Bureau of Standards, Washington, 1964.

[3] G. P. Ivantsov, Dokl. Akad. Nauk SSSR 58 (1947) 567.

[4] D.A. Kessler, H. Levine, Phys. Rev. A 36 (1987) 4123.

[5] D.A. Kessler, H. Levine, Acta metall. 36 (1988) 2693.

[6] M. Ben Anıar, E. Brener, Phys. Rev. Lett. 71 (1993) 589.

[7] E. Brener, V.I. Mel'nikov, JETP 80 (1995) 341.

[8] J. C. LaCombe, M. B. Koss, V. E. Fradkov, M. E. Glicksman, Phys. Rev. E. 52 (1995) 2778 .

[9] M. E. Glicksman, M. B. Koss, L. T. Bushnell, Jeffrey C. LaCombe, E. A. Winsa, ISIJ Int. 35 (1995) 604.

[10] M.B. Koss, L.T. Bushnell, J.C. LaCombe, M. E. Glicksman, Chem. Eng. Comm. 152153 (1996) 351.

[11] M.E. Glicksman, N.B. Singh, in Rapidly Solidified Powder Aluminum Alloys, ed. M.E. Fine and E.A. Starke, Jr. (ASTM, Philadelphia, 1986), p. 44.

[12] M. Muschol, D. Liu, H.Z. Cummins, Phys. Rev. A 46 (1992) 1038.

[13] J.W. Cahn D.W. Hoffman, Acta Metall. 22 (1974) 1205.

[14] P.W. Voorhees, S.R. Coriell, G.B. McFadden, R.F. Sekerka, J. Crystal Growth 67 (1984) 425.

[15] S.R. Coriell, G.B. McFadden, Morphological Stability, in Handbook of Crystal Growth, Vol. 1B, ed. D. T. J. Hurle, (Elsevier, Amsterdam, 1993), pp. 785-857.

[16] A. Karma, W.-J. Rappel, Phys. Rev. E 57 (1998) 4342.

[17] J.C. LaCombe, Ph. D. Thesis, Department of Materials Science and Engineering, Rensselaer Polytechnic Institute, Troy, NY, December, 1998.

[18] V. Pines, A. Chait, M. Zlatkowski, J. Crystal Growth 167 (1996) 383.

[19] R.F. Sekerka, S.R. Coriell, G.B. McFadden, J. Crystal Growth 171 (1997) 303. 


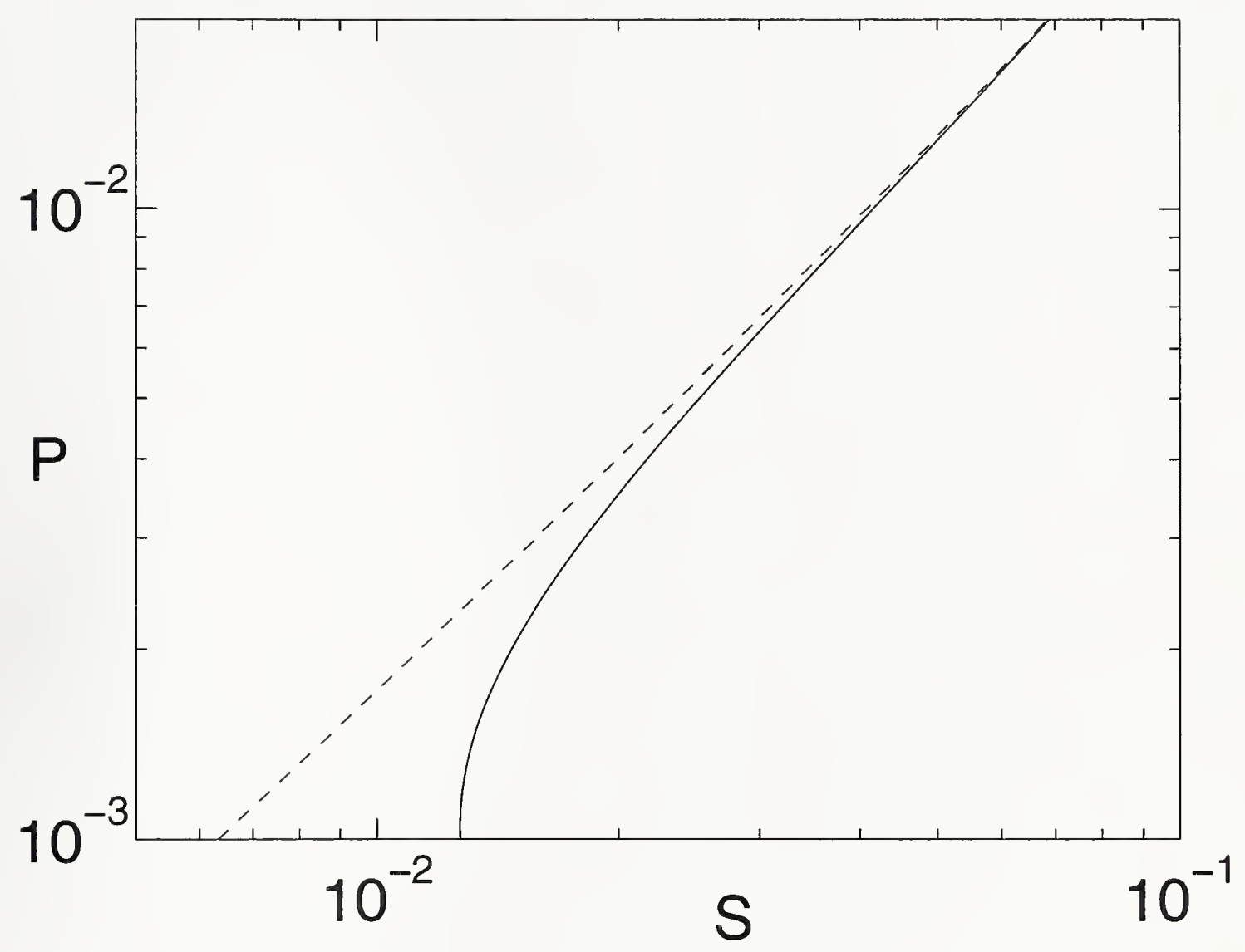

Figure 1: The Peclet number $P$ as a function of dimensionless supercooling $S$ for shape parameter $\epsilon=-0.008$ (solid curve) and $\epsilon=0$ (dashed curve); the dashed curve corresponds to the Ivantsov solution. 

